\title{
Contextual optimization of location-based routing protocols for multi-hop cellular networks using mobile relays
}

\author{
B. Coll-Perales · J. Gozalvez
}

Received: date / Accepted: date

\begin{abstract}
Traditional single-hop cellular architectures fail to provide high and homogeneous quality of service levels throughout a cell area due to the strong signal attenuation with the distance. In this context, multi-hop cellular networks that utilize mobile relays and device-to-device communications have been proposed to overcome the physical limitations of conventional cellular architectures. One of the key building blocks of multi-hop cellular networks is the multi-hop routing of information from the source to the destination. This paper investigates the performance and energy signaling benefits of location-based multi-hop routing protocols. In particular, the paper demonstrates the benefits of a contextual optimization of this type of protocols in order to achieve a high end-to-end performance, while reducing the energy and signaling implementation cost.
\end{abstract}

Keywords Multi-hop Cellular Networks · Multihop routing - Mobile relays - Device-to-Device communications $\cdot$ Contextual optimization $\cdot 5 \mathrm{G}$

\section{Introduction}

Cellular networks have significantly evolved over the past decades with the emergence of new and efficient radio access technologies, and the implementation of advanced techniques. This evolution has mainly focused around the tra-

B. Coll-Perales $(\bowtie) \cdot J$. Gozalvez

Uwicore, Ubiquitous Wireless Communications Research Laboratory University Miguel Hernndez of Elche

Avda. de la Universidad, s/n, 03202, Elche, Spain

Tel.: +34 965222031

Fax: +34966658903

E-mail: bcoll@umh.es

J. Gozalvez

E-mail: j.gozalvez@umh.es ditional infrastructure-centric architecture where each Mobile Station (MS) directly communicates with the Base Station (BS). Despite the considerable efforts made in the research community, traditional single-hop cellular architectures fail to provide high and homogeneous Quality of Service (QoS) levels throughout the cell area (in particular at the cell edges) due to the strong signal attenuation with the increasing distance. Such signal degradations result in that mobile users located at the cell boundaries experience poor QoS levels. This problem could be partially overcome by augmenting the number of BSs, although provisioning additional infrastructure bears significant costs at the deployment and management phases, and it has also a social cost. Other emerging alternatives currently being considered as part of $5 \mathrm{G}$ network evolution include the direct communication between devices or Device-to-Device communications (D2D), and the integration of cellular and ad-hoc or D2D communications into what is referred to as Multi-hop Cellular Networks with Mobile Relays (MCN-MR) [20,24]. MCN-MR networks are characterized by a lower implementation cost than the deployment of additional infrastructure, but a higher management complexity due to the participation of mobile devices. However, exploiting the mobile devices' networking and communications capabilities in a decentralized and distributed manner also increases the potential and future perspectives of MCN-MR networks. To reach such potential, it is necessary to overcome important technological challenges, such as the design and optimization of robust, adaptive, context-aware and energy-efficient multi-hop routing protocols.

Analytical and simulation-based studies have reported the benefits and advantages that MCN-MR networks can provide over traditional cellular architectures in terms of capacity, coverage, infrastructure deployment cost, power saving and energy efficiency ([4]). First experimental studies have recently demonstrated the gains achieved by MCN-MR 
networks with regards to energy efficiency, end-user QoS and link quality when operating at large distances to the serving BS, in indoor environments, and under Non-LineOf-Sight (NLOS) propagation conditions [9]. These gains result from the substitution of long-distance (and usually NLOS) single-hop cellular links by shorter distance (LOS) links with improved link budgets. Field tests have also demonstrated how MCN-MR networks using D2D communications can improve the end-user QoS in cell-overlaid or handover areas [7]. In the conducted experimental studies, multi-hop MCN-MR links experience good LOS conditions and short distances among communicating nodes. In addition, the multi-hop path between the BS and the destination MS is predefined at the start of each field trial. In this context, important technological challenges need yet to be overcome, such as the design and optimization of efficient and robust multi-hop routing protocols.

Wireless mesh technologies, such as those based on the IEEE 802.11 s standard $[1,12]$, are suitable candidates for the multi-hop ad-hoc operation of MCN-MR networks since they incorporate novel and innovative networking functions capable to improve the performance and operation of self-organized and distributed networks. One of these functions, that is also crucial for MCN-MR networks, is the multi-hop routing. Routing protocols are in charge of selecting the relaying nodes over which a packet will be transmitted from the source to the destination. The multihop routing protocol included in the IEEE 802.11s standard tends to achieve a good performance at the expense of a significant overhead in the route search process. This overhead can result in potential high channel congestion levels, and significant energy consumption dedicated solely to signaling proposes [6]. These constraints difficult it use in multi-hop cellular networks employing mobile terminals as relaying nodes.

To improve the operation and performance of multihop routing protocols, several studies have proposed the use of location information to limit the search area for a new multi-hop route in order to minimize the signaling overhead, and therefore the routing energy consumption. One of the first and most relevant location-aided proposals, the LAR (Location-Aided Routing) protocol [15], limits the area where route request messages are propagated based on the assumption of the knowledge of the position and average speed of the destination node. However, the LAR protocol does not take into account during its multi-hop route search process the communication environment, the radio propagation conditions, or the potential mobile terminal's limitations e.g. in terms of battery. Subsequent contributions have aimed to reduce the LAR routing overhead by using directional antennas (Directional Antenna Multipath LAR with On Demand Transmission Power, DA-MLARODTP) [8], creating baselines between the source and desti- nation and selecting the next relaying node based on the distance to the baseline (Improved LAR, ILAR) [19], adding back-up routes (LAR With Back-up, LARWB) [13], or tracing the change of distance between nodes (Distance Based LAR, DBLAR) [16]. Although interesting, all these proposals are based on the LAR protocol, and it is important to note that they thereby inherit its routing search philosophy, which is based on minimizing the time to establish a multi-hop route, and does not consider the state of the network and of the potential relaying nodes. Other alternative multi-hop routing protocols exploiting location information are greedy forwarding techniques, such as GPSR (Greedy Perimeter Stateless Routing) [14], that have been investigated both for MANETs (Mobile Ad-hoc NETworks) and VANETs (Vehicular Ad-hoc NETworks) [10]. These techniques route packets from the source to the destination by selecting forwarding nodes that are closest to the destination. To this aim, greedy forwarding techniques require each node to periodically broadcast its position in beacon messages. To reduce the risk of link failures due to the selection of forwarding nodes that are at the limit of the radio range, the GOLI (Greedy On-demand using Location Information) [18] proposal defines a radio range threshold, and selects the next hop node based on this threshold. It is important to note that greedy techniques base their operation only on the knowledge of the state of 1-hop neighbors, and forward packets towards the destination without previously creating a multi-hop route from the source to the destination. This results in that there is no guarantee of the delivery of the forwarded packets to the destination node, which in turn could result in very inefficient and useless multi-hop transmissions. These drawbacks limit the applicability of these proposals to MCN-MR networks that require the provision of adequate QoS levels, while efficiently using the communications channel and reducing the signaling overhead. In addition, the use of mobile terminals as relaying nodes requires the design of multi-hop routing protocols that minimize their energy consumption in order to foster their cooperation in the relaying process [22]. To achieve these objectives, this paper proposes and optimizes a multi-hop routing protocol that addresses the needs of MCN-MR networks by exploiting the knowledge of the location of the destination node. As this study will demonstrate, the configuration of the proposed protocol can be adapted to its operating environment in order to improve its efficiency without sacrificing its end-to-end QoS performance.

\section{IEEE 802.11s ad-hoc networking}

This work is based on the ad-hoc networking capabilities of the IEEE 802.11s amendment [12], which is developed to add mesh functionalities to the IEEE 802.11 standard (the concepts proposed in this paper could be applied to other 
technologies). To this aim, IEEE 802.11 s creates a Wireless Distribution System (WDS) with automatic topology learning and wireless path configuration. In particular, the IEEE 802.11s standard defines new networking functions such as mesh discovery process, mesh route establishment, channel selection, mesh links management, congestion control, authentication and security. The mesh network discovery process is enabled through the periodic broadcast exchange of beaconing messages among neighboring nodes. Beacon messages contain different information elements such as the Service Set IDentifier (SSID), Mesh ID, Mesh Configuration, etc., that ensure the interoperability between mesh nodes. In addition, beacon messages contain reserved fields to meet special future application needs.

The routing protocol proposed in the IEEE 802.11s standard is the Hybrid Wireless Mesh Protocol (HWMP), which includes both a reactive and proactive operational mode. This work is focused on the reactive modified version of the AODV (Ad-hoc On-Demand Distance Vector) protocol that is part of HWMP. AODV [21] is a reactive routing protocol that only searches and establishes a route from the source to the destination when the source has information to transmit, and does not know the route to reach the destination node. In this case, the source node sends a broadcast Route REQuest (RREQ) message that is retransmitted by neighboring nodes. When the destination node receives the RREQ message, it replies with a unicast Route REPly (RREP) message to confirm the route establishment. The reception of the RREQ and RREP messages allows intermediate nodes to know their neighboring nodes in the route towards the source and destination nodes respectively. In the original AODV protocol, the route selected between the source and destination nodes is that with the lower latency, which generally coincides with the route with the lowest number of hops from the source to the destination. In this sense, the intermediate and destination nodes discard RREQ packets generated in the same route search process (detected with a sequence number) that have already been processed. Contrary to the original AODV, the modified version proposed in the IEEE 802.11s standard allows intermediate nodes to retransmit a RREQ packet more than once if the new route reduces the established multi-hop route cost function, compared to the previously selected multi-hop route. Therefore, while the original AODV protocol tends to select the route with the lowest latency, the modified AODV version selects the route that optimizes the selected cost function. The cost of the multi-hop route is updated along the discovery path by means of adding the cost of each of the links. This allows intermediate nodes to make local decision on the necessity of retransmitting the RREQ packets. To compute the cost of the multi-hop link, IEEE 802.11s includes a default mandatory path selection metric (Airtime Link) that aims to reflect the amount of channel resources consumed by transmitting a test frame over a particular link. According to [12], the Airtime Link metric is computed as follows:

$C_{a}=\left[O+\frac{B_{t}}{r}\right] \frac{1}{1-e_{f}}$

where $O$ is a constant overhead latency that varies according to the physical layer implementation, $B_{t}$ is the test frame size ( 8192 bits), $r$ is the data rate in $\mathrm{Mb} / \mathrm{s}$ at which the mesh node would transmit a test frame, and $e_{f}$ is the measured test frame error rate. Apart from the default Airtime Link metric, IEEE 802.11s also allows the implementation of additional cost functions designed for special application needs, or intended for specific networking requirements. The requirements for designing efficient routing metrics in wireless mesh networks are summarized in [2].

\section{Multi-hop routing protocols for MCN-MR networks}

MCN-MR networks using mobile terminals as relaying nodes require networking mechanism ensuring adequate QoS levels while efficiently using the nodes resources and the communication channel. Despite its high performance, the HWMP routing protocol defined in the IEEE 802.11s standard is not an adequate technique for MCN-MR networks due to its significant signaling cost in the route search process (as previously explained, the modified version of AODV allows intermediate nodes to retransmit an RREQ packet more than once). To overcome the signaling, and consequently energy inefficiencies of HWMP, the authors have proposed an energy-efficient multi-hop routing protocol tailored for MCN-MR networks, and that bases its operation on the knowledge of the location of the destination node [5]. Following this first proposal, this work investigates how to optimize the protocol's operation to reduce its energy and signaling impact without sacrificing its end-to-end QoS performance. The authors' proposal bases its operation on the path selection metric defined by L. Cao et al. in [3] since it considers some of the most relevant parameters for the operation of MCN-MR networks.

\subsection{Multiple-Metric routing protocol}

Different multi-hop routing protocols and cost functions have been proposed in the literature for wireless mesh networks. Most of them are based on the number of hops from the source to the destination, although additional proposals have also considered parameters such as the energy, the network congestion, the packet error rate or the throughput in the multi-hop route search process [11]. In this context, the Multiple-Metric (MM) proposal [3] has been selected as the reference technique over which to compare the multi-hop routing protocol proposed in this paper, due 
to its performance, and the fact that its cost function is based on some of the most relevant parameters (energy, channel congestion and number of hops from the source to the destination) for the performance and operation of MCNMR networks. In addition, the MM proposal was also based on the modified AODV routing protocol proposed in the IEEE $802.11 \mathrm{~s}$ standard. The MM multi-hop routing protocol selects the multi-hop route from the source to the destination that minimizes the following cost function:

cost $=\alpha_{1}$ hops $+\alpha_{2}$ load $+\alpha_{3}$ energy

with hops representing the number of hops from the source to the destination, load the channel congestion, and energy the node's energy consumption. The $\alpha$ variables are defined to weight the importance of the three parameters in the MM cost function. In our implementation, $\alpha$ has been chosen to ensure that all parameters vary between 0 and 1 . The definition or implementation of these parameters slightly differs to that used in [3] due to the consideration of different technologies and objectives. In particular, the channel congestion is estimated in the MM implementation using the time between two data packets, since MM considers Constant Bit Rate (CBR) traffic. This approach is not feasible for bursty data traffic, therefore the channel congestion is here estimated using the beacon messages that are regularly transmitted in the IEEE 802.11s standard. In the absence of channel congestion, a node would periodically receive the beacon messages from a neighboring node. As the channel congestion increases, the reception of the beacon messages can be delayed, with the delay increasing with the channel congestion. Given that the beacon's periodicity is known by all nodes, this work estimates the channel congestion using the beacon's message reception delay. Therefore, the channel congestion estimate is a consequence of the MAC layer's contention process when transmitting beacon messages. In this case, the time interval between beaconing messages (int $v l)$ is estimated as:

int $v l=\frac{t_{\text {now }}-t_{\text {last }_{\text {beacon }}}-t_{\text {beacon }}}{t_{\text {beacon }}} ; 0<$ int $v l<1$

with $t_{\text {now }}, t_{\text {last }_{\text {beacon }}}$ and $t_{\text {beacon }}$ representing the current time, the time at which the last beacon was received and the beacon's periodicity, respectively. The load is then estimated considering the previous load estimate (load $\left._{\text {previous }}\right)$ as follows:

load $=(1-\beta)$ load $_{\text {previous }}+\beta$ int $v l$

with the $\beta$ parameter offering the possibility to differently weight the importance of the load previous $_{\text {and }}$ int $v$ l estimates, which allows to take into account the dynamic and unpredictable properties of the wireless channel and avoids considering the last channel load estimate as the reference measure. For the energy factor, the initial MM proposal was targeted at minimizing the transmission power cost. On the other hand, in this work the energy factor represents the energy consumed by the mobile node and it is aimed at maximizing the nodes' lifetime battery by trying to distribute the energy consumption over all the mobile nodes of the network. This is the case because the energy factor favors the selection of intermediate mobile nodes with higher energy levels to cooperate in the communication between the source and the destination nodes. As shown in equation (5), the energy factor is calculated as the difference between the node's initial energy $\left(E_{\text {init }}\right)$ and the node's current energy $\left(E_{\text {now }}\right)$ :

energy $=E_{\text {init }}-E_{\text {now }}$

The normalization of the weight of the energy factor in the cost function has been achieved by setting the $\alpha_{3}$ parameter equal to $1 / E_{\text {init }}$. Finally, the influence of the number of hops in the multi-hop route selection process is here considered by normalizing the number of hops to a maximum number or hops $s_{\max }$. If this maximum is exceeded, the hops factor is set equal to 1, but routes are not restricted to hops $s_{\max }$ hops. Normalizing the hops factor is aimed at avoiding an overweight of this parameter in the cost function when selecting the multi-hop route from the source to the destination.

\section{$3.2 x$ hops Location permissive-Multiple Metric}

The authors showed that modifying the IEEE $802.11 \mathrm{~s}$ multihop route search process, by preventing intermediate nodes that are further away from the destination node than the previous relaying node to retransmit RREQ messages, can reduce the network flooding and the energy consumption [5]. However, these improvements come at the expense of reducing the application packet delivery ratio due to a decrease in the number of candidate relaying nodes. The $x$ hops Location permissive-Multiple Metric ( $x$ LoMM) technique is proposed to trade-off performance and cost of multi-hop routing protocols. $x$ LoMM is based on the IEEE 802.11s reactive multi-hop route search process, but limits the network flooding by exploiting the knowledge of the position of the destination and candidate relaying nodes. The position of relaying nodes is known from the periodic beacon messages broadcasted by IEEE 802.11s mobile nodes. Originally, $x$ LoMM allows any node to retransmit a received broadcast RREQ message only if the node is nearer to the destination node than the node from which it received the RREQ message. However, MCN-MR networks are characterized by the mobility of relaying nodes and the challenging radio propagation conditions that difficult the reliable transmission of information between peer mobile terminals. These characteristics could strongly limit the number of candidate relaying nodes that guarantee the progress 
towards the destination node, thereby decreasing the endto-end multi-hop connectivity and performance. In this context, the $x$ LoMM proposal provides the capacity to tradeoff multi-hop end-to-end connectivity and signaling/energy cost by allowing a maximum of $x$ relaying nodes that are not in the direction towards the destination node. To this aim, $x$ LoMM incorporates the ' $x$ permissions' field in the RREQ packet, which indicates how many additional relaying nodes that do not progress towards the destination node are allowed before a multi-hop route is discarded. In addition, $x$ LoMM identifies all candidate multi-hop routes using the MM cost function. The difference with the original MM technique is that the number of candidate multi-hop routes is significantly reduced with $x \mathrm{LoMM}$, and so is the signaling and energy cost of establishing a multi-hop connection.

Fig. 1 illustrates the operation of the $x$ LoMM proposal (Fig. 2 shows the $x$ LoMM's flow chart). In the example, the source node $(\mathrm{S})$ launches a multi-hop route search process to reach the destination node (BS) allowing a maximum of 2 relaying nodes $(x=2)$ that do not represent a progress towards the destination node. Although $\mathrm{MS}_{1}$ is further away from BS than node $S$, it is allowed to retransmit the RREQ packet at the expense of decreasing the ' $x$ permissions' field to $1 . \mathrm{MS}_{2}$ is then selected as a relaying node to retransmit the RREQ packet received from $\mathrm{MS}_{1}$ without reducing the ' $x$ permissions' field since it is closer to the BS than node $\mathrm{MS}_{1}$. From $\mathrm{MS}_{2}$, the RREQ packet is relayed through $\mathrm{MS}_{3}, \mathrm{MS}_{4}$ and $\mathrm{MS}_{5}$ nodes. However, the fact that only a maximum of 2 relaying nodes that do not progress towards the destination node are allowed in a multi-hop route search process by the $x$ LoMM technique will ensure that the multi-hop route search process is stopped at node $\mathrm{MS}_{6}$. As a result, $x$ LoMM reduces the network flooding and energy consumption of relaying terminals by intelligently limiting the multi-hop route search process through the use of location information. However, a key aspect yet to be defined, and that is investigated in this paper, is what is the optimum setting

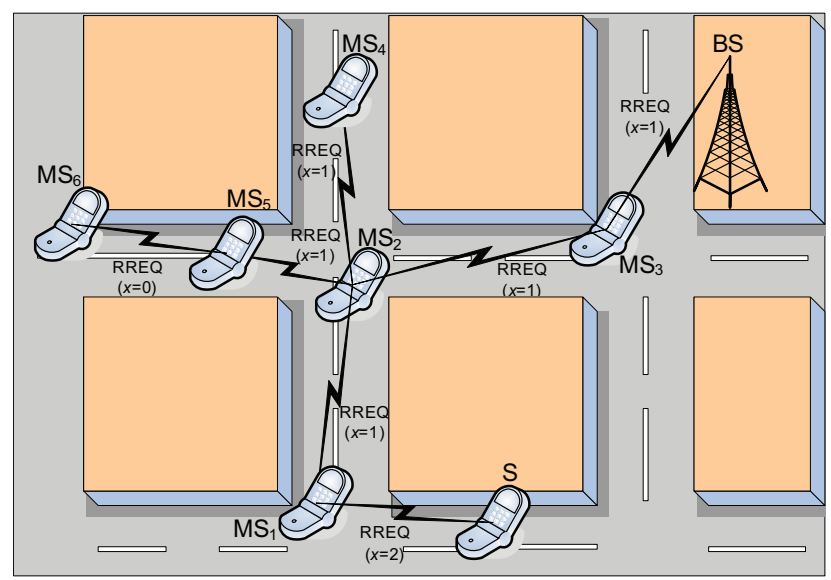

Fig. 1 Operation of $x$ LoMM

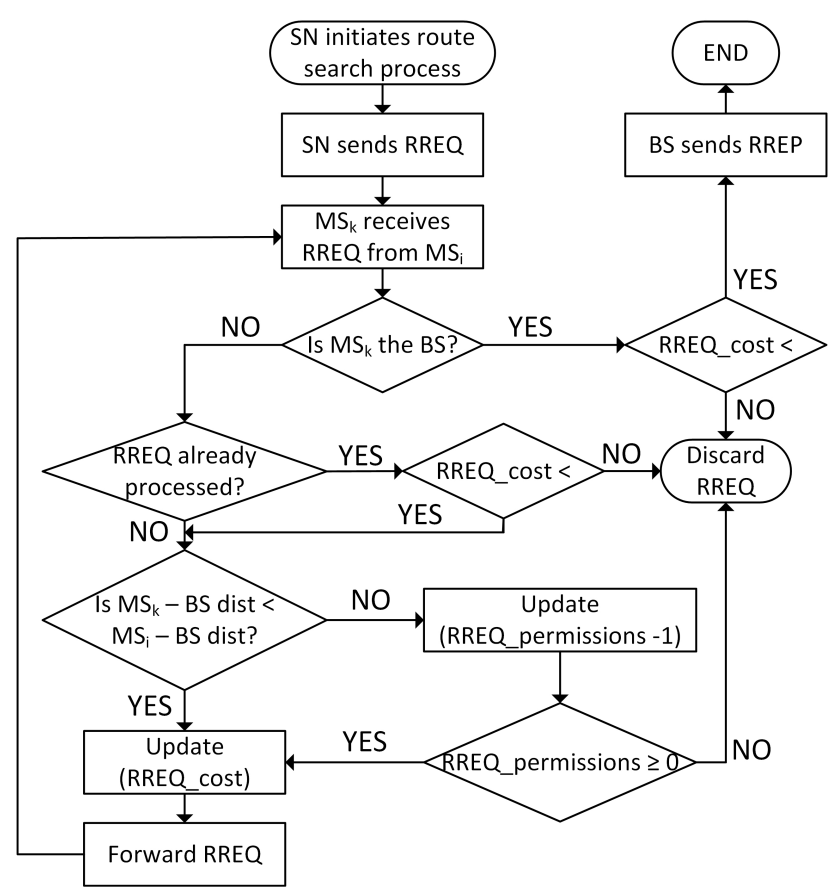

Fig. 2 Graphical representation of the operation of $x$ LoMM

of the ' $x$ permissions' parameter that enables reducing the signaling and energy cost of multi-hop transmissions without sacrificing the end-to-end performance or packet delivery ratio. As this work will demonstrate, a contextaware selection of this parameter, based on the source node's location and the cellular network characteristics, offers the capability to optimize the performance and implementation cost of the $x$ LoMM proposal.

\section{Evaluation environment}

To investigate the performance of the proposed multi-hop routing protocol, this study is based on a Manhattan type scenario emulated with the ns2.29 software platform. A Base Station (BS) is located at the centre of the scenario, and nodes move following the 'Random Walk Obstacle' mobility model [17]. The study focuses on a scenario composed of a single BS, but the extension to a multi-BS scenario could be feasible as long as the information that the proposed $x$ LoMM protocol needs to establish a multihop route (i.e. the location of the target BS) is updated when mobile nodes pass from one cell to another. This study considers uplink transmissions, and assumes that the position of the destination node (BS) is known. This assumption is in fact realistic since, for example, many countries have currently open databases with the location of all their base stations. Although this study initially focuses on uplink transmissions, an adaptation to downlink communications could also be feasible. In this case, the position of the destination node would be extracted through 
Table 1 NS2 simulation parameters

\begin{tabular}{ll}
\hline Parameter & Value \\
\hline Node density (nodes/m) & 0.01 \\
Building width (m) & 200 \\
Street width (m) & 25 \\
Transmission power (W) & 0.2 \\
Transmission rate (Mbps) & 12 \\
Node's speed (m/s) & 1.5 \\
Data packet size (bytes) & 500 \\
Beacon's period (s) & 1 \\
hops $_{\text {max }}$ & 15 \\
\hline
\end{tabular}

the use of GPS devices and the source node (the BS) could be informed of this position using the signaling capabilities of cellular networks.

Nodes communicate using the IEEE 802.11a technology at $5.8 \mathrm{GHz}$ and the propagation loss is modelled through the WINNER pathloss model developed for urban microcellular scenario [23]. This model distinguishes between Line of Sight (LOS) and Non-Line of Sight (NLOS) propagation conditions, which is of paramount importance in this work due to the presence of the obstructing buildings. To the authors's knowledge, this model is one of the most suitable for studying MCN-MR networks since it reproduces urban environment with relatively low BS antenna heights. An "on-off" bursty pattern traffic model has been implemented in order to simulate 200 seconds traffic sessions, with "on" and "off" periods lasting for 5 and 15 seconds respectively. The selected "off" period ensures that the nodes routing table validity has expired at the start of the following "on" period. This results in that for each "on" period at least one route search process is launched. During the "on" period, the source nodes transmit 50 packets to the BS. The traffic sessions are generated following a Pareto distribution, and multiple users can act as traffic sources simultaneously. Each simulation run-time is 10.000 $\mathrm{s}$ and the obtained results are average values of multiple experiments. The remaining simulation platform parameters are summarized in Table $1^{1}$.

\section{$5 x$ LoMM performance}

The performance of the $x$ LoMM proposal is compared against that achieved using the MM technique. The MM technique does not limit the route search process, which results in a high Packet Delivery Ratio (PDR) at the cost of a significant network flooding and signaling energy consumption. The optimization process of the $x$ LoMM

\footnotetext{
1 The value of hops $s_{\max }$ has been chosen to realize large multi-hop connections (not necessarily limited to hops $s_{\max }$ hops). However, since realistic multi-hop transmissions would probably employ a reduced number of hops, other values of hops $s_{\max }$ could be feasible.
}

proposal should then target to achieve a comparable PDR to the original MM technique, while significantly reducing the signaling overhead and energy consumption. To analyze the impact of the operating environment on the optimum setting of the $x$ LoMM proposal, this work considers three different cellular scenarios: macro-cells with 1590 m cell radius, macro-cells with $950 \mathrm{~m}$ cell radius and micro-cells with 320 $\mathrm{m}$ cell radius. The node density in each of these scenarios (Table 1) has been chosen to ensure that the MM technique achieves a similar PDR performance (92\%), which therefore represents a similar probability to find a multi-hop route from the source to the destination.

It is important to note that the larger the cell radius, the larger the average distance between the source (MS) and destination (BS) nodes, and the higher the number of hops necessary to establish an end-to-end connection. Also, as the number of hops increases, the probability to successfully establish a multi-hop route also decreases. Consequently, in the case of the $x$ LoMM proposal, it could be expected that higher values of the ' $x$ permissions' field are necessary to achieve the PDR performance of the MM proposal as the cell radius increases. In addition, it is important to note that in urban environments, the larger the distance between the source and destination nodes, the higher the probability of having to find relaying nodes at intersections. These intersections can limit the number of forwarding nodes that progress towards the destination due to the potential operation under NLOS propagation conditions. An example is illustrated in Fig. 3, where $S_{1}$ cannot establish a direct link to $R_{2}$ to forward its packet to the destination $B S$ due to the presence of the building. Instead, the node $S_{1}$ should

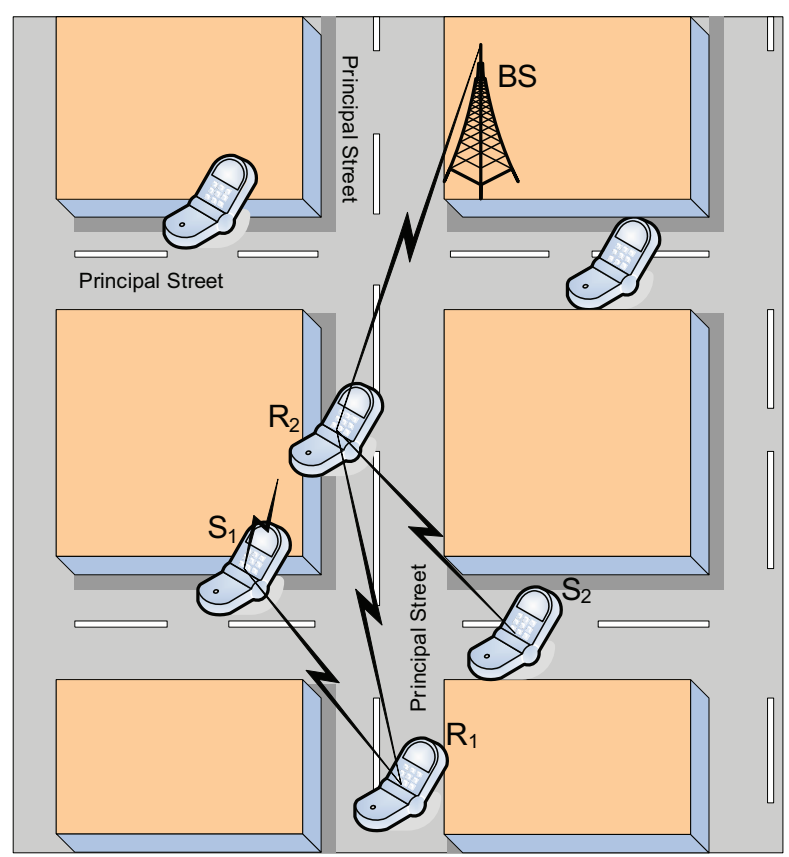

Fig. 3 Corner effect for the $x$ LoMM proposal 
first relay the packet to $R_{1}$ and then to $R_{2}$. In the case of the $x$ LoMM proposal, this relaying process will consume ' 1 permission' given that $\mathrm{R}_{1}$ is further away from $\mathrm{BS}$ than the source node $\mathrm{S}_{1}$.

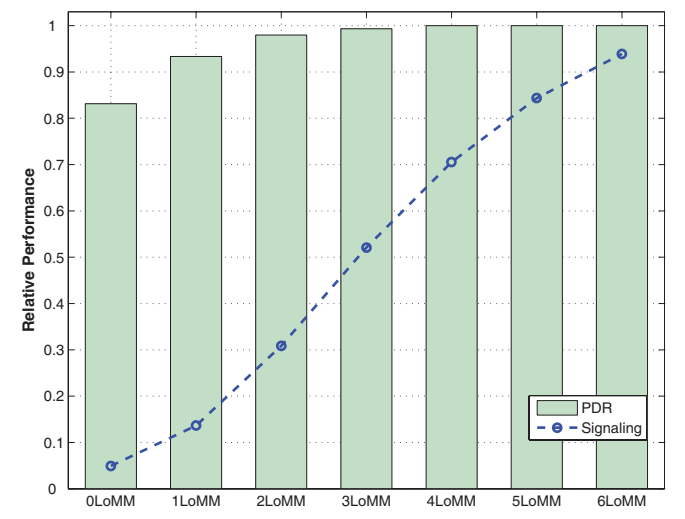

(a) Macro-cell scenario with $950 \mathrm{~m}$ cell radius

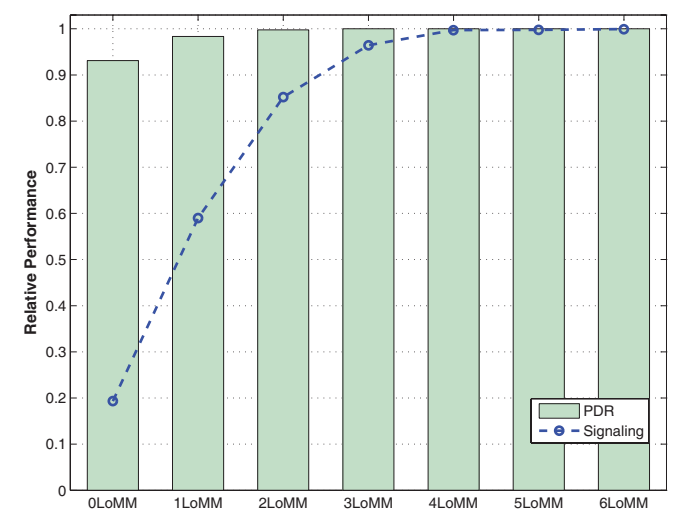

(b) Micro-cell scenario with $320 \mathrm{~m}$ cell radius

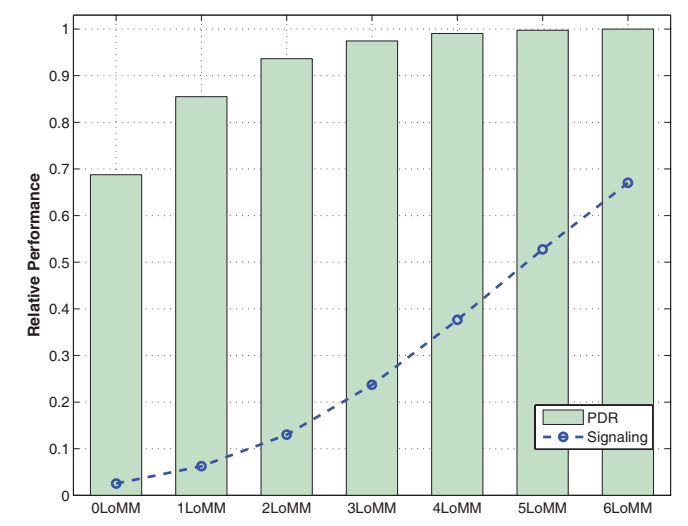

(c) Macro-cell scenario with $1590 \mathrm{~m}$ cell radius

Fig. $4 x$ LoMM PDR performance and signaling cost relative to MM
Fig. 4a shows for the macro-cell scenario of $950 \mathrm{~m}$ cell radius, the ratio of the PDR and signaling cost obtained with the $x$ LoMM proposal to that experienced with the MM protocol. The signaling cost is measured as the number of RREQ messages retransmitted by relaying nodes in the process to establish a multi-hop route from the source to the destination. The results shown in Fig. 4a highlight that if no permissions are granted in the $x$ LoMM technique (OLoMM), its performance cannot increase beyond $85 \%$ of PDR achieved with MM. This is due to the fact that OLoMM implementation only considers relaying nodes that progress towards the destination node, and if such relaying nodes are not available the route from the source to the destination cannot be established (Fig. 3), and the source node drops its data packet. Of course, limiting the multi-hop route search area significantly reduces the signaling cost of the $x$ LoMM proposal, but the results shown in Fig. 4a demonstrate the need to grant a certain number of permissions if a PDR performance close to MM is targeted. However, the results illustrated in Fig. 4a clearly emphasize that a limited number of permissions is sufficient to achieve such performance, while still offering the capacity to reduce the routing signaling cost. For example, with just '2 permissions' in the route search process, 2LoMM is capable to achieve $95 \%$ of the MM PDR performance, while reducing the signaling load by $70 \%$. The same performance as MM is obtained with ' 4 permissions', while still reducing the signaling load by $30 \%$. Increasing the permissions beyond 4 in the considered cellular deployment scenario does not further improve the performance with respect to $\mathrm{MM}$, but increases the signaling load. As shown in Fig. 5a, reducing the signaling load reduces the energy consumption due to the multi-hop route search process. For example, the $4 \mathrm{LoMM}$ technique that achieves the same PDR performance as MM while reducing the signaling load by $30 \%$, results in a decrease of $25 \%$ in terms of the energy consumed in the multi-hop route search process (ERouting). ERouting represents the ratio of the mean energy consumption per node derived from all the tasks needed to establish a multi-hop route (e.g transmission and reception of RREQ and RREP packets) measured with the $x$ LoMM proposal with respect to MM. Fig. 5a also shows the relative energy consumption derived from the transmission and reception of data packets (EData), and the relative average total energy consumption per node (ETotal); ETotal does not account for the beaconing energy consumption that is equal to all nodes. All these values are in relative terms, which explains why ETotal is not equal to the sum of all energies. It is very important to note that on average the $4 \mathrm{LoMM}$ proposal achieving the same PDR performance as MM is capable to reduce the total energy consumption of relaying nodes by $15 \%$.

Finally, Table 2 compares some of the main characteristics of the multi-hop routes established using the $x$ LoMM 
Table 2 Multi-hop operation for the macro-cell scenario with $950 \mathrm{~m}$ cell radius

\begin{tabular}{llllll}
\hline Technique & \# hops & $\begin{array}{l}\text { Route_d } \\
{[\mathrm{m}]}\end{array}$ & $\begin{array}{l}\text { Thr_app } \\
{[\mathrm{Mbps}]}\end{array}$ & $\begin{array}{l}\text { RTT } \\
{[\mathrm{ms}]}\end{array}$ & \% Broken \\
\hline MM & 4.66 & 823.19 & 2.25 & 19.20 & 24.12 \\
0LoMM & 3.83 & 691.03 & 2.56 & 15.20 & 17.24 \\
1LoMM & 4.17 & 743.29 & 2.38 & 17.10 & 21.39 \\
2LoMM & 4.40 & 785.64 & 2.29 & 18.10 & 23.30 \\
3LoMM & 4.56 & 815.50 & 2.26 & 18.50 & 23.32 \\
4LoMM & 4.64 & 820.57 & 2.25 & 18.80 & 23.58 \\
5LoMM & 4.65 & 822.77 & 2.25 & 18.80 & 23.71 \\
6LoMM & 4.65 & 823.19 & 2.25 & 19.20 & 23.98 \\
\hline
\end{tabular}

and MM techniques in the macro-cell scenario with cell radius equal to $950 \mathrm{~m}$. It is interesting to observe that limiting the relaying nodes search area (i.e. reducing the number of permissions in the $x$ LoMM operation) reduces the number of hops from the source to the destination (\#hops) and consequently the total multi-hop route distance (Route_d ), and the time needed to find an optimum route from the source to the destination, measured in terms of the Round Trip Time $(R T T)$. These values increase as the number of permissions granted to $x$ LoMM increases, and finally reaches a value similar to that obtained with MM. The convergence to the MM performance was also observed for the PDR, and consequently the throughput at the application layer (Th_app). It is important to note that although OLoMM improves the throughput performance, this is only done for the established multi-hop routes, and the number of established multi-hop routes is lower for OLoMM than for other $x$ LoMM implementations as reflected in the PDR performance (Fig. 4a). Despite the convergence of its performance with $\mathrm{MM}$ as the number of permission increases, $x$ LoMM always guarantee more stable and robust multi-hop routes, measured in terms of percentage of broken multi-hop routes during a data session with respect to the total number of established multihop routes (\%Broken).

\section{Impact of the cellular deployment scenario}

As it has been shown in the results discussed so far, the $x$ LoMM operation can be optimized through the adequate setting of the ' $x$ permissions' field that enables reaching a performance equal to that obtained with the reference MM technique, while significantly reducing the signaling load, or network flooding, and the consequent energy consumption of the relaying nodes. It is interesting now to investigate whether the optimal setting of $x$ LoMM should depend on the contextual operating environment, for example, in terms of the cellular deployment characteristics. In this context, Fig. $4 \mathrm{~b}$ shows for the micro-cell scenario of 320 $\mathrm{m}$ cell radius, the ratio of the PDR and signaling cost obtained with the $x$ LoMM proposal to that experienced with
Table 3 Multi-hop operation for the micro-cell scenario with $320 \mathrm{~m}$ cell radius

\begin{tabular}{llllll}
\hline Technique & \# hops & $\begin{array}{l}\text { Route_d } \\
{[\mathrm{m}]}\end{array}$ & $\begin{array}{l}\text { Thr_app } \\
{[\mathrm{Mbps}]}\end{array}$ & $\begin{array}{l}\text { RTT } \\
{[\mathrm{ms}]}\end{array}$ & \% Broken \\
\hline MM & 2.19 & 305.27 & 5.09 & 6.50 & 15.67 \\
0LoMM & 1.85 & 243.71 & 5.38 & 4.70 & 10.87 \\
1LoMM & 2.05 & 287.94 & 5.16 & 5.80 & 13.85 \\
2LoMM & 2.13 & 300.34 & 5.10 & 6.30 & 14.69 \\
3LoMM & 2.18 & 305.20 & 5.09 & 6.50 & 15.34 \\
4LoMM & 2.18 & 305.22 & 5.09 & 6.50 & 15.59 \\
5LoMM & 2.18 & 305.25 & 5.09 & 6.50 & 15.59 \\
6LoMM & 2.18 & 305.25 & 5.09 & 6.50 & 15.59 \\
\hline
\end{tabular}

the MM protocol. A comparison of Figs. 4a and $4 \mathrm{~b}$ shows that the $x$ LoMM proposal achieves a better performance for a given number of permissions under a more aggressive cellular deployment scenario due to the closer distance between mobile nodes and the BS. In fact, 1LoMM and 2LoMM achieve $95 \%$ and $100 \%$ of the MM PDR performance respectively in the micro-cell scenario, whereas ' 4 permissions' were needed in the macro-cell scenario with $950 \mathrm{~m}$ cell radius to achieve the same performance as MM. These results clearly emphasize the need to adapt the setting of the ' $x$ permissions' field to the specific cellular deployment scenario in order to guarantee the highest possible performance while reducing the signaling load, and consequently the energy consumption in the multi-hop route search process (see Fig. 5b, in particular the ERouting parameter). As shown in Fig. $5 b$, the optimum setting of the ' $x$ permissions' field that allows reaching the same performance as MM (2LoMM) in the micro-cell scenario, enables reducing by $5 \%$ the average total energy consumption of relaying nodes compared to when operating with MM.

In terms of the operation of the proposed techniques in a micro-cellular deployment scenario, the results shown in Table 3 confirm that the $x$ LoMM proposal results in a lower number of hops from the source to the destination, and thereby a lower RTT and total multi-hop route distance, as a consequence of limiting the multi-hop route search process using the knowledge of the destination's location. As previously highlighted, $x$ LoMM also increases the throughput at the application level and the robustness of the multi-hop routes, although its performance converges to that achieved with MM as the number of permissions increases.

To conclude the study on the optimum performance and energy consumption setting of the $x$ LoMM proposal as a function of the cellular deployment characteristics, Fig. $4 \mathrm{c}$ shows, for the macro-cell scenario of $1590 \mathrm{~m}$ cell radius, the ratio of the PDR and signaling cost obtained with the $x$ LoMM proposal to that experienced with the MM protocol. The comparison of Figs. $4 \mathrm{a}, 4 \mathrm{~b}$ and $4 \mathrm{c}$ shows that as the average distance between mobile nodes and base station increases, the $x$ LoMM technique needs 


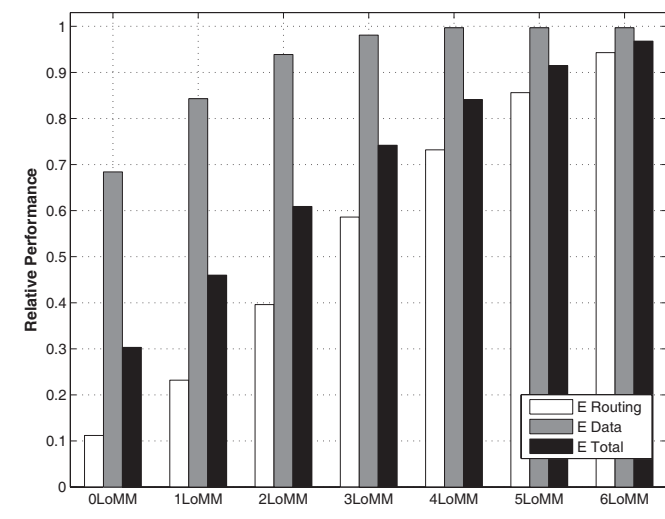

(a) Macro-cell scenario with 950 m cell radius

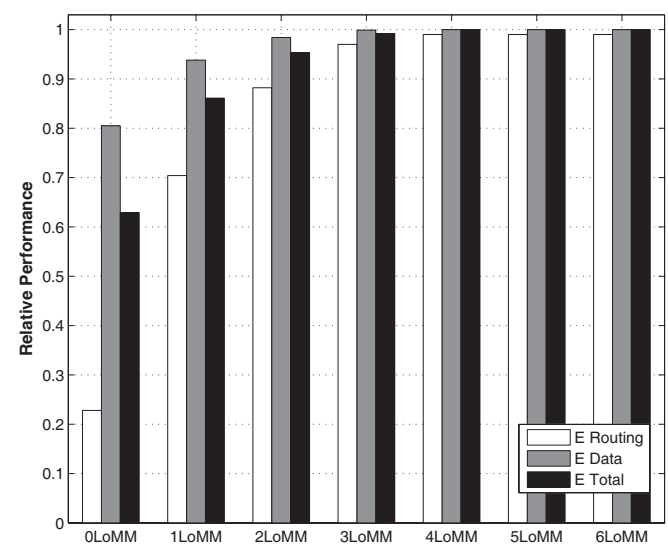

(b) Micro-cell scenario with 320 m cell radius

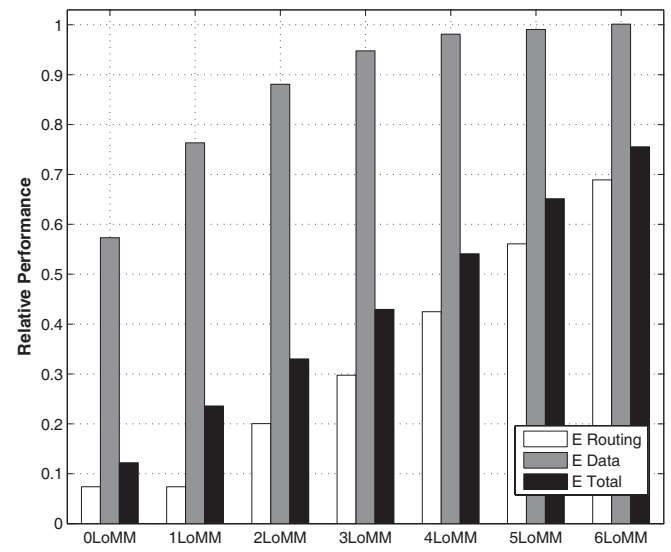

(c) Macro-cell scenario with 1590 m cell radius

Fig. 5 LLoMM energy consumption relative to MM

to increase its ' $x$ permissions' value to obtain the same PDR performance as MM. In the macro-cell scenario with $1590 \mathrm{~m}$ cell radius, the ' $x$ permissions' field has to be increased to reach the same performance as MM. However,
Table 4 Multi-hop operation for the macro-cell scenario with $1590 \mathrm{~m}$ cell radius

\begin{tabular}{llllll}
\hline Technique & \# hops & $\begin{array}{l}\text { Route_d } \\
{[\mathrm{m}]}\end{array}$ & $\begin{array}{l}\text { Thr_app } \\
{[\mathrm{Mbps}]}\end{array}$ & $\begin{array}{l}\text { RTT } \\
{[\mathrm{ms}]}\end{array}$ & \% Broken \\
\hline MM & 7.91 & 1436.2 & 1.13 & 33 & 30.61 \\
OLoMM & 6.45 & 1194.5 & 1.38 & 27 & 28.25 \\
1LoMM & 6.93 & 1275.9 & 1.25 & 29 & 29.14 \\
2LoMM & 7.23 & 1335.2 & 1.18 & 30 & 30.01 \\
3LoMM & 7.50 & 1366.7 & 1.15 & 31 & 30.21 \\
4LoMM & 7.64 & 1405.3 & 1.14 & 32 & 30.47 \\
5LoMM & 7.75 & 1410.5 & 1.13 & 32 & 30.60 \\
6LoMM & 7.76 & 1417.6 & 1.13 & 32 & 30.61 \\
\hline
\end{tabular}

it is interesting to note that the larger the average distance to the destination node (or cell radius), the higher the signaling reduction achieved with the $x$ LoMM performance. In fact, the 5LoMM technique achieving the same PDR performance as MM reduces by nearly 50\% the signaling load, whereas the reduction achieved with the 2LoMM and 4LoMM techniques in the micro-cellular and macro-cellular scenario with $950 \mathrm{~m}$ cell radius was equal to $15 \%$ and $30 \%$ respectively. This significant signaling reduction notably decreases the energy consumption of mobile nodes (Fig. 5c). In fact, the results depicted in Fig. 5c show that the 5LoMM technique achieving the same PDR performance as MM in the macro-cell scenario with $1590 \mathrm{~m}$ cell radius reduces by $45 \%$ the multi-hop route search process energy consumption of relaying nodes (ERouting) compared to MM. This results in that the total energy consumption of relaying nodes operating with the 5LoMM proposal is just $60 \%$ of that consumed when operating with the MM proposal, which clearly emphasizes the important signaling and energy benefits of the $x$ LoMM proposal without sacrificing the endto-end performance. In terms of the multi-hop operation of the techniques under evaluation, similar trends (see Table 4) as those described for the previous cellular deployment scenarios are also obtained for the macro-cellular scenario with 1590 m cell radius.

\section{Impact of the location of the source node}

The previous section has demonstrated the energy and signaling benefits of a contextual adaptation of the $x$ LoMM proposal. To further optimize (both in terms of performance and energy consumption) the operation of the $x$ LoMM proposal, this section investigates the use of additional contextual data, in particular the knowledge of the source node's location. In this context, three different location areas have been identified: Line of Sight (LOS), intersection and street. In the LOS area, source nodes are located under LOS propagation conditions with the destination BS. Source nodes located in intersection areas have LOS propagation conditions with four streets or road segments, two of which 
progress towards the destination, which increases the route search diversity. Source nodes located in the street area do not have LOS conditions with the destination node (as LOS area), and do not benefit from the route diversity experienced in the intersection area. As a result, these nodes have more challenging communication conditions to reach the destination node.

Fig. 6a shows the ratio of the PDR and signaling cost obtained with the $x$ LoMM proposal to that experienced with the MM protocol for the macro-cell scenario with $950 \mathrm{~m}$ cell radius. The results illustrated in Fig. 6 differentiate the performance based on the location of the source node. As it can be observed from Fig. 6a, source nodes with LOS conditions to the BS only need ' 0 permissions' to achieve the same performance as MM, while reducing the multi-hop route search process signaling overhead by $97 \%$. Consequently, the OLoMM technique applied to source nodes located in the LOS area also results in an average total energy consumption (ETotalLOS) of only $25 \%$ of that consumed by the MM proposal (see Fig. 7a). Source nodes located at intersection areas can benefit from the mentioned route diversity to achieve the same performance as MM with only ' 3 permissions', compared to users in the street area that require the ' 4 permissions' highlighted in Fig. $4 \mathrm{a}$. It is also important to note that the source nodes located at the intersection area generate more multi-hop signaling load due to the route diversity that offers the proposed technique the possibility to propagate the route search process among two streets progressing towards the destination. Although such diversity increases the end-to-end performance compared to the case in which the source nodes are located in the street area, it also results in a higher energy consumption (Fig. 7a). In any case, it is important to highlight that the energy benefits of the $x$ LoMM proposal are maintained independently of the source's node location, and that such benefits are reduced as the number of permissions increases. These results show that using the knowledge of the location of the source node, the operation and performance of the $x$ LoMM proposal can be further optimized to achieve the same PDR performance as MM, while reducing the signaling load and energy consumption. The performance, signaling and energy differences based on the location of the source node are also observed in the micro-cellular scenario (Figs. 6b and 7b). For the micro-cell scenario, Fig. $7 \mathrm{~b}$ shows that the ratio of the ETotalLOS energy consumption achieved with the OLoMM proposal to that experienced with the MM technique does not follow the trends shown in the other scenarios under study. This is simply due to the shorter distances between the source and destination nodes experienced in this specific scenario. Such short distances allow delivering a higher percentage $(30 \%)$ of data packets directly to the BS without the execution of the route search process when the source nodes are located

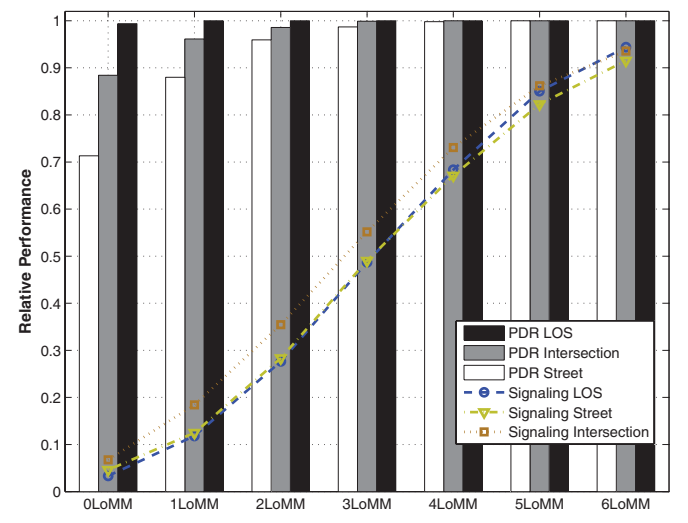

(a) Macro-cell scenario with $950 \mathrm{~m}$ cell radius

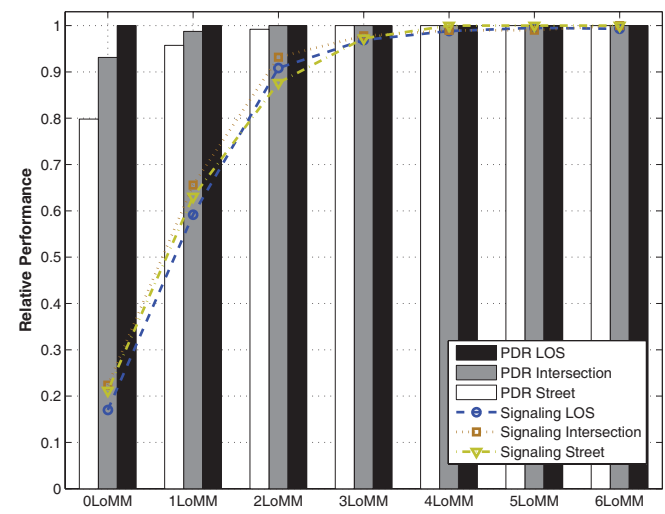

(b) Micro-cell scenario with $320 \mathrm{~m}$ cell radius

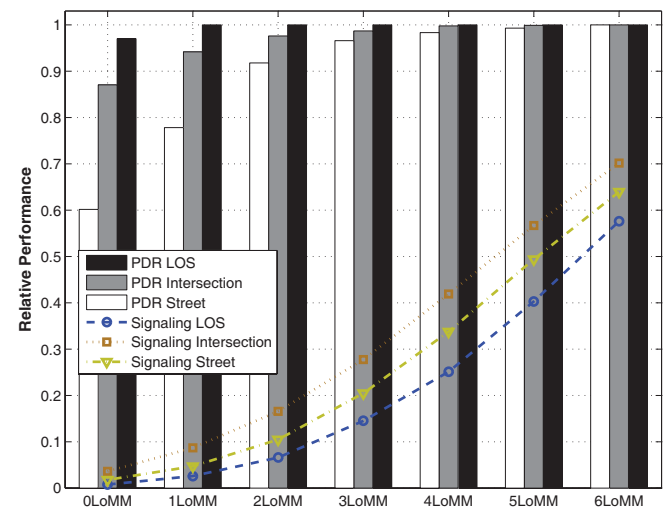

(c) Macro-cell scenario with $1590 \mathrm{~m}$ cell radius

Fig. $6 x$ LoMM PDR performance and signaling cost relative to MM based on the source node's location

in the LOS area. In this context, the energy consumed by MM and OLoMM is quite similar, which explains the higher ratio of the ETotalLOS energy consumption compared to the case in which source nodes are located at intersection or street areas. Finally, it is important to highlight that the 
performance differences between MM and $x$ LoMM, and thereby the benefits achieved by exploiting the source node's location, are higher as the cell radius increases (as it can be appreciated in Figs. 6c and 7c).

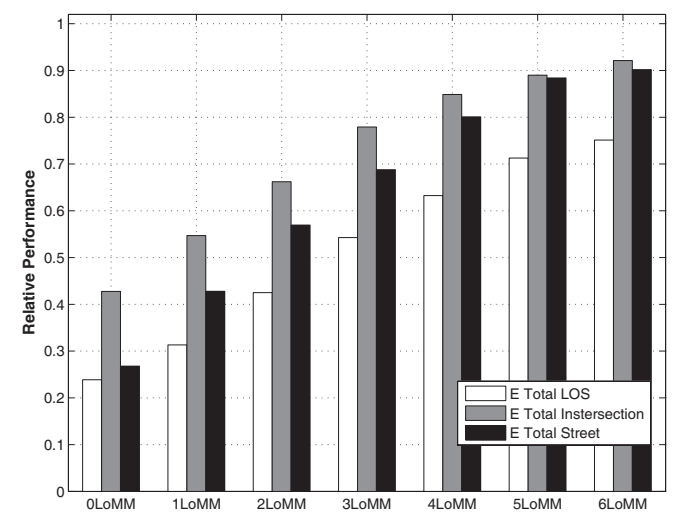

(a) Macro-cell scenario with $950 \mathrm{~m}$ cell radius

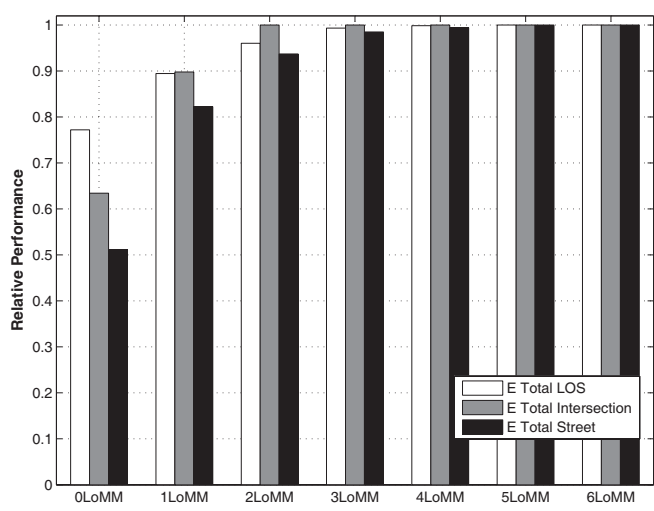

(b) Micro-cell scenario with $320 \mathrm{~m}$ cell radius

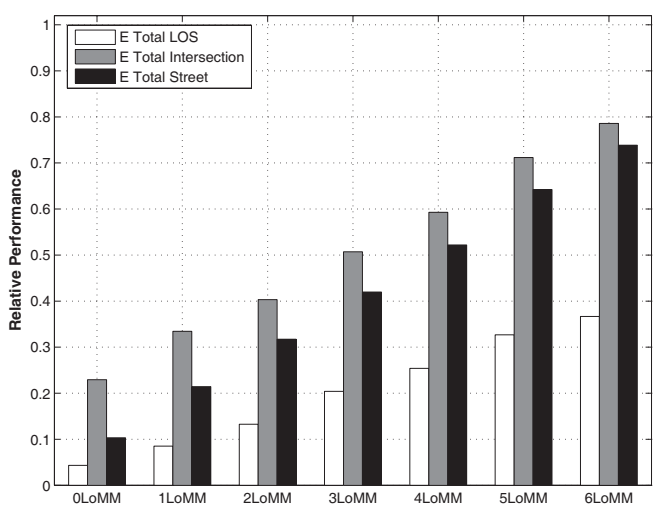

(c) Macro-cell scenario with 1590 m cell radius

Fig. $7 x$ LoMM total energy consumption relative to MM based on the source node's location

\section{Conclusions}

This work has proposed and investigated the use of novel multi-hop routing protocols for MCN-MR networks. The proposed technique exploits the location information to reduce the signaling and energy consumption of relay nodes without sacrificing its end-to-end performance. In addition, this work has demonstrated that the performance of the proposed location-based multi-hop routing protocol can be further optimized based on contextual information, such as the cellular deployment characteristics, or the location of the source node. Using this contextual data, the proposed technique can significantly reduce the energy consumption of mobile nodes, which is a crucial factor for a potential future deployment of $\mathrm{MCN}$ networks based on mobile relays.

Acknowledgements This work has been supported by the Ministry of Economy and Competitiveness (Spain) and FEDER funds under the projects TEC2008-06728 and TEC2011-26109, by the Generalitat Valenciana under the project ACOMP/2010/111 and ACIF/2010/161, and by the Ministry of Industry, Tourism and Trade (Spain) under the project TSI-020400-2008-113 (CELTIC proposal CP5-013).

\section{References}

1. Akyildiz, I.F., \& Wang, X. (2009). Wireless mesh networks, advanced texts in communications and networking. 1st ed. Wiley.

2. Ashraf, U., Abdellatif, S., \& Juanole, G. (2011). Route selection in IEEE 802.11 wireless mesh networks. Telecommunication Systems Journal, 52(4), 1-19.

3. Cao, L., Sharif, K., Wang, Y., \& Dahlberg, T. (2008). Adaptive multiple metrics routing protocols for heterogeneous multi-hop wireless network. Proceedings of the 5th IEEE Consumer Communications \& Networking Conference (CCNC), pp. 13-17, USA.

4. Cavalcanti, D., Agrawal, D., Cordeiro, C., Bin, X., \& Kumar, A. (2005). Issues in Integrating Cellular Networks, WLANs, and MANETs: a Futuristic Heterogeneous Wireless Network. IEEE Wireless Communications, 12(3), 30- 41.

5. Coll-Perales, B., \& Gozalvez, J. (2009). Energy efficient routing protocols for multi-hop cellular networks. Proceedings of the 20th IEEE Personal Indoor and Mobile Radio Communications (PIMRC), pp. 1457-1461, Japan.

6. Coll-Perales, B., \& Gozalvez, J. (2009). Neighbor selection techniques for multi-hop wireless mesh networks. Proceedings of the 9th IEEE Workshop on Wireless Local Networks (WLN), pp. 10201026, Switzerland.

7. Coll-Perales, B., \& Gozalvez, J. (2011). On the capability of multihop cellular networks with mobile relays to improve handover performance. Proceedings of the 8th International Symposium on Wireless Communication Systems (ISWCS), pp. 207-211, Germany.

8. Gajurel, S., Malakooti, B., \& Limin, W. (2007). DA-MLAR-ODTP: directional antenna multipath location aided routing with on demand transmission power. Proceedings of the 2nd IEEE International Symposium on Wireless Pervasive Computing (ISWPC), pp. 1-6, Puerto Rico.

9. Gozalvez, J., \& Coll-Perales, B. (2013). Experimental evaluation of multihop cellular networks using mobile relays. IEEE Communications Magazine, 51(7), pp. 122-129.

10. Gozalvez, J., Sepulcre, M., \& Bauza, R. (2010). Impact of the radio channel modelling on the performance of VANET 
communication protocols. Telecommunication Systems Journal, 119. Doi: 10.1007/s11235-010-9396-x.

11. Iannone, L., \& Fdida, S. (2006). Evaluating a cross-layer approach for routing in wireless mesh networks. Telecommunication Systems Journal, 31(2), 173-193.

12. IEEE Std 802.11s (2011). Amendment to IEEE Std 802.11: mesh networking. IEEE Standard.

13. Kalhor, S., Anisi, M., \& Haghighat, A.T. (2007). A new positionbased routing protocol for reducing the number of exchanged route request messages in mobile ad-hoc networks. Proceedings of the 2nd IEEE International Conference on Systems and Networks Communications (ICSNC), pp.13-19, France.

14. Karp, B., \& Kung, H.T. (2000). GPSR: greedy perimeter stateless routing for wireless networks. Proceedings of the 6th ACM International Conference on Mobile Computing and Networking (MobiCom), pp. 243-254, USA.

15. Ko, Y., \& Vaidya, H. (2000). Location-aided routing (LAR) in mobile ad-hoc networks. Wireless Networks, 6(4), 307-321.

16. Kun, W., \& Meng, W. (2009). DBLAR: a distance-based locationaided routing for MANET. Journal of Electronics (China), 6(2), 152-160.

17. Maeda, K., Uchiyama, A., Umedu, T., Yamaguchi, H., \& Higashino, T. (2009). Urban pedestrian mobility for mobile wireless network simulation. Ad Hoc Networks, 7(1), 153-170.

18. Nakagawa, H., Ishida, K., Ohta, T., \& Kakuda, Y. (2006). GOLI: greedy on-demand routing scheme using location information for mobile ad hoc networks. Proceedings of the 26th IEEE International Conference on Distributed Computing Systems Workshops (ICDCS), pp. 1- 6, Portugal.

19. Nen-Chung, W., \& Si-Ming, W. (2005). An efficient locationaided routing protocol for mobile ad-hoc networks. Proceedings of the 11th IEEE International Conference on Parallel and Distributed Systems (ICPADS), pp. 335-341, Japan.

20. Pabst, R., et al (2004). Relay-based deployment concepts for wireless and mobile broadband radio. IEEE Communication Magazine, 42(9), 80-89.

21. Perkins, C., \& Royer, E. (1999). Ad-hoc on-demand distance vector routing. Proceedings of the 2nd IEEE Workshop on Mobile Computing Systems and Applications (WMCSA), pp. 90-100, USA.

22. Rodriguez-Mayol, A., \& Gozalvez, J. (2014). Reputation based selfishness prevention techniques for mobile ad-hoc networks. Telecommunication Systems Journal, 57(2), 181-195.

23. WINNER. D1.1.1. WINNER II interim channel models. Public deliverable, https://www.ist-winner.org/index.html.

24. Zhou, J., Venkatesha Prasad, R., Lu, Y., \& Niemegeers, I. (2013). Simulation-based analysis of a multi-hop integrated UMTS and WLAN network. Telecommunication Systems Journal, 52 (1), 327 340 . 